\title{
EDESONFILARIA CYNOCEPHALI n. sp., Filaire parasite de Dermoptère en Malaisie.
}

\author{
0. BAIN*, B. KOUYATE* et J. CASSONE*
}

RÉSUMÉ. Description d'Edesonfilaria cynocephali n. sp., parasite de Cynocephalus variegatus taylori (Thomas) en Malaisie. Mise en synonymie de Makifilaria Krishnasamy et coll., 1981 avec Edesonfilaria et formation de la nouvelle combinaison E. inderi (Krishnasamy et coll., 1981) n. comb. Edesonfilaria et le genre très proche Macacanema constituent une petite lignée de Filaires à œsophage hyperspécialisé (portion glandulaire dépourvue de lumière); elle est propre à la région indo-malaise et parasite de Dermoptères, Chiroptères et Primates arboricoles.

\section{Edesonfilaria cynocephali n. sp., Filarial parasite of a Dermopteran in Malaisia.}

SUMMARY. Edesonfilaria cynocephali n. sp., a parasite of Cynocephalus variegatus taylori (Thomas) in Malaysia, is described. Makifilaria Krishnasamy et coll., 1981 is placed in synonymy with Edesonfilaria and the new combination E. inderi (Krishnasamy et coll., 1981) n. comb. is proposed. Edesonfilaria and the closely related genus Macacanema constitute a small evolutionary line of Filariae with a hyperspecialized oesophagus (the glandular portion lacks lumen); the line is restricted to the Indo-Malaysian region and occurs in arboreal Dermopterans, Chiropterans and Primates.

Des Filaires récoltées en $1974 \mathrm{chez}$ un Cynocephalus variegatus taylori (Thomas) dans l'île malaise de Pulau Tioman nous ont été très aimablement remises par le Dr. Lim Boo Liat, de l'Institute for Medical Research de Kuala Lumpur.

Ces Filaires constituent une nouvelle espèce qui se rattache au petit groupe Macacanema - Edesonfilaria, remarquable par la structure très particulière de l’œsophage.

* Laboratoire des Vers, associé au CNRS, Muséum National d'Histoire Naturelle, 61 rue de Buffon, F 75231 Paris Cedex 05.

I. Ce travail a été effectué grâce à une subvention de l'Organisation Mondiale de la Santé. Accepté le II octobre I982. 


\section{Matériel}

1 옹olype, $1 \hat{\sigma}$ allotype, 2 우 et $1 \hat{\sigma}$ paratypes (lot 167 JE, M.N.H.N. Paris).

\section{Description ( $f$ ig. 1 et 2)}

Filaires très larges et transparentes. Tête avec 8 papilles accolées 2 à 2 et disposées suivant un rectangle étiré latéralement. Papilles céphaliques en forme de petites languettes ovales; pores des amphides très petits. Bouche petite ; cavité buccale réduite, simplement limitée par la cuticule céphalique épaissie ; œsophage divisé ; la portion musculaire se prolonge le long de la portion glandulaire en un tube à paroi mince, d'aspect feuilleté en coupe transversale et qui devient de plus en plus réduit jusqu'à se terminer en cul-de-sac à environ $1 \mathrm{~mm}$ du début de l'œsophage glandulaire. La portion glandulaire elle-même, est dépourvue d'une lumière œesophagienne ; elle apparaît formée, après analyse histologique (colorations à l'Unna-Brachet et action de la ribonucléase), par un syncitium long d'une douzaine de mm, aux noyaux peu nombreux et périphériques et à la masse centrale cytoplasmique remplie de grains de ribosomes ; ce syncitium est entouré par une lame basale épaisse ; à la fin de l'œsophage, la lame basale forme 3 mamelons qui font saillie dans l'intestin. L'intestin a un aspect normal avec une lumière axiale. L'interruption du canal digestif au niveau de l'œsophage glandulaire entraîne un cheminement particulier des substances nutritives absorbées avec 2 passages au travers de membranes : un premier passage à travers la paroi de l'œsophage musculaire vers le syncitium glandulaire, un $2^{\mathrm{e}}$ passage à travers la lame basale épaisse qui ferme ce syncitium vers la lumière intestinale. Les noyaux du syncitium glandulaire, très volumineux, à 1 ou 2 gros nucléoles vacuolaires, et l'abondance des ribosomes suggèrent une grande activité métabolique de cet organe, où sont vraisemblablement transformés les aliments ingérés. Queue courte et arrondie, ornée par un tubercule subterminal de forme irrégulière.

\section{Femelle}

Vulve près du début de l'œsophage glandulaire ; vagin rectiligne à sphincter constitué par 3 larges cellules renflées; ovéjecteur long, presque droit suivi d'une poche utérine impaire très longue; ovaires minces, rectilignes; opistodelphie.

Microfilaires utérines : ni gaine, ni corps interne ; corps aminci aux deux extrémités ; tête séparée du corps par un cou légèrement marqué ; pointe caudale anucléée.

Dimensions : Femelle holotype : corps long de $90 \mathrm{~mm}$ et large de $780 \mu \mathrm{m}$; anneau nerveux à $170 \mu \mathrm{m}$ de l'apex; papilles de la tête disposées suivant un rectangle de $70 \mu \mathrm{m}$ sur $35 \mu \mathrm{m}$; œsophage long de $11,8 \mathrm{~mm}$ avec portion glandulaire débutant à $700 \mu \mathrm{m}$ de l'apex; vulve à $1070 \mu \mathrm{m}$ de l'apex ; portion impaire du tube génital longue de 5,62 $\mathrm{mm}$; ensemble ovaire-oviducte long de 12,3 $\mathrm{mm}$; queue longue de $45 \mu \mathrm{m}$. 

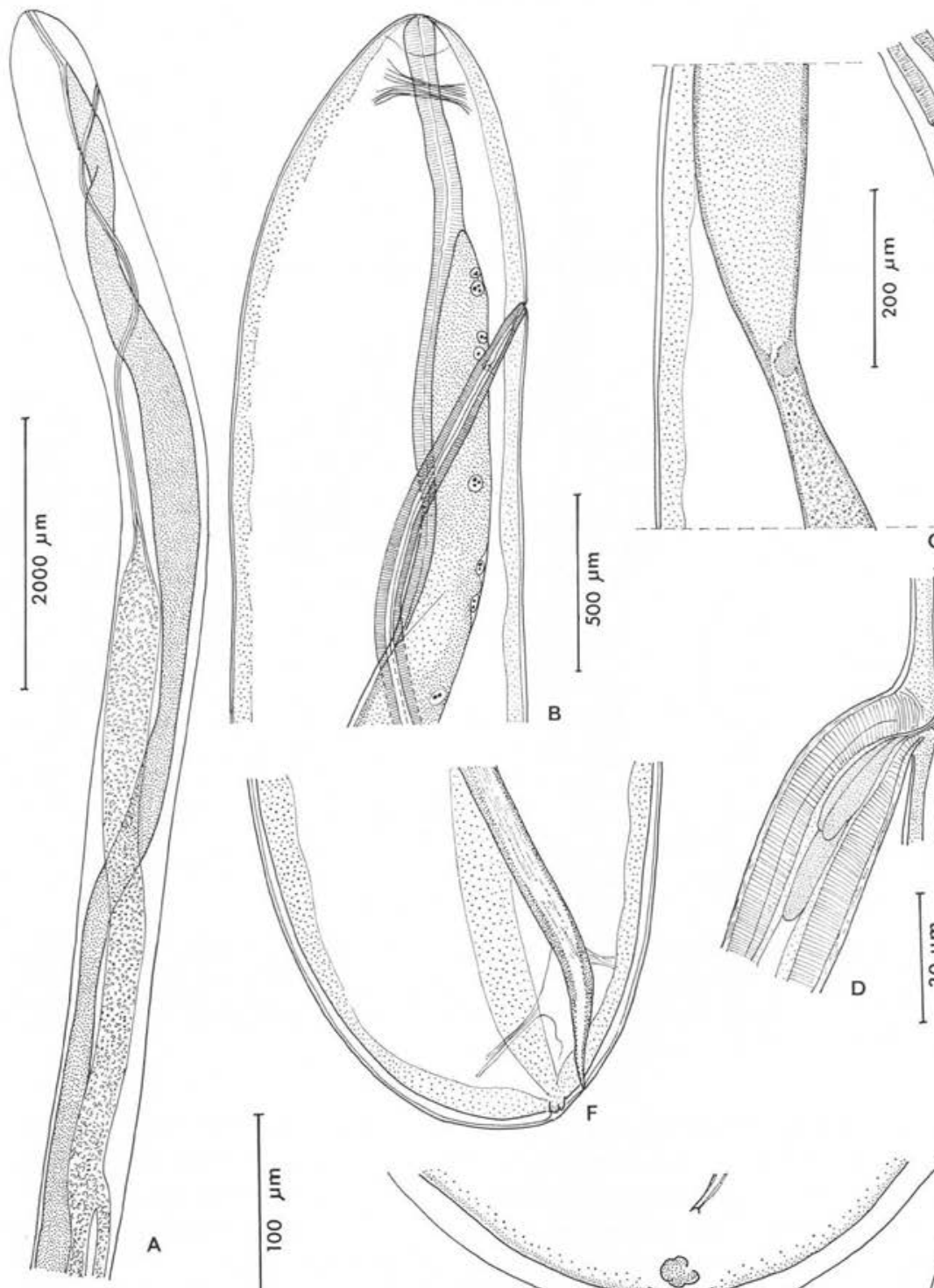


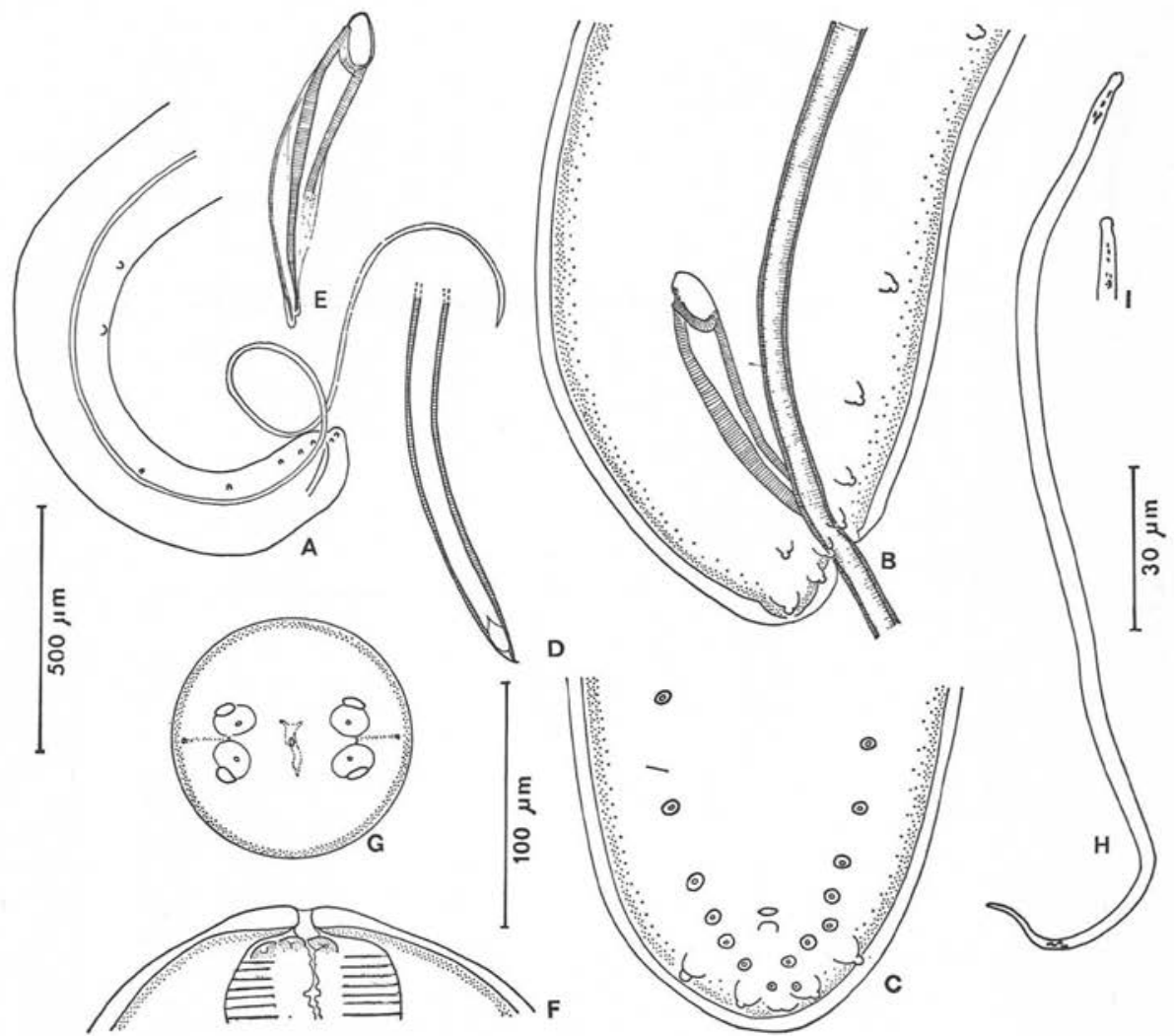

FIG. 2. - Edesonfilaria cynocephali n. sp., A à F, mâle; A : région postérieure, vue latérale ; $\mathrm{B}$ : région caudale, vue latérale ; $\mathrm{C}$ : idem, vue ventrale; $\mathrm{D}$ : extrémité du spicule gauche, vue ventrale ; E : spicule droit, vue latérale ; F : tête, vue latérale; G à I, femelle; G : tête, vue apicale; $\mathrm{H}$ : microfilaire utérine, vue latérale ; I : tête, vue médiane (A éch. $500 \mu \mathrm{m}$; B, C, D, E, F, G éch. roo $\mu \mathrm{m}$; H, I éch. $30 \mu \mathrm{m})$.

Une femelle paratype : corps long de $78 \mathrm{~mm}$ et large de $680 \mu \mathrm{m}$; anneau nerveux à $200 \mu \mathrm{m}$ de l'apex; œsophage long de $16,3 \mathrm{~mm}$ avec portion glandulaire débutant à $600 \mu \mathrm{m}$ de l'apex; vulve à $820 \mu \mathrm{m}$ de l'apex; portion impaire du tube génital longue de $11,3 \mathrm{~mm}$; ovaire-oviducte long de $11 \mathrm{~mm}$; queue longue de $50 \mu \mathrm{m}$.

Microfilaires utérines longues de 190-212 $\mu \mathrm{m}$ et larges de 3,5 $\mu \mathrm{m}$.

\section{Mâle}

Papilles caudales : 5 paires précloacales sur 2 files latéroventrales, 4 paires postcloacales sur 4 files, dont 2 presque latérales, 2 à 4 paires de papilles surnuméraires étagées en avant des papilles précloacales ; phasmides entre les papilles de la dernière paire postcloacale. Spicule gauche filiforme extrêmement long ; spicucle droit court, épais en forme de gouttière. 

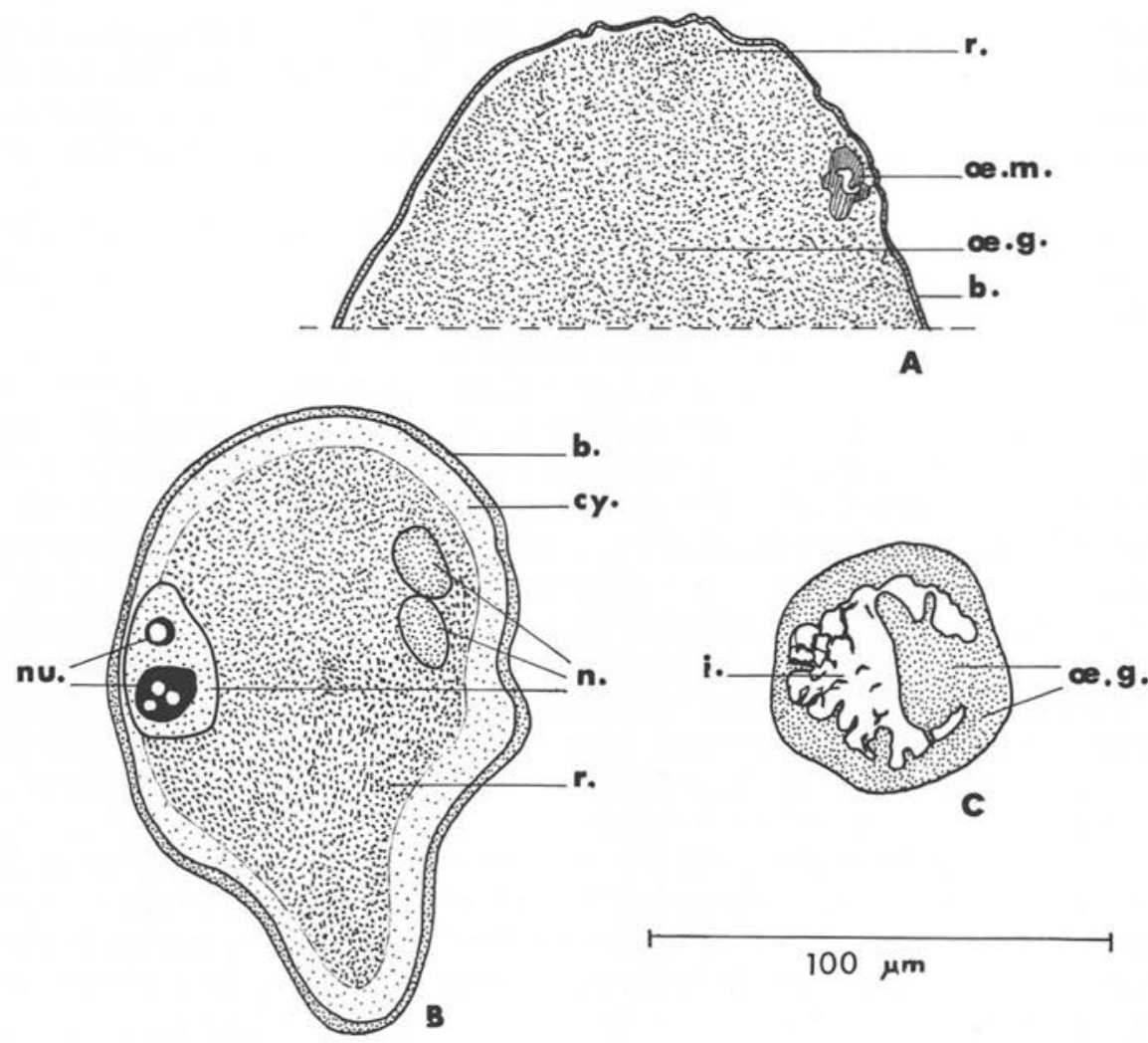

FIG. 3. - Edesonfilaria cynocephali $\mathrm{n}$. sp., structure de l'œesophage, coupes histologiques transversales ; A : fin de l'œsophage musculaire ; B : région postérieure de l'œsophage glandulaire ; $\mathrm{C}$ : jonction œsophage glandulaire (lame basale hypertrophiée) et intestin (structure vacuolaire) (éch. roo $\mu \mathrm{m})$; b. : lame basale ; cy. : cytoplasme ; i. : intestin ; n. : noyau ; nu. : nucléole; $œ . m$. : œsophage musculaire ; œ.g. : œsophage glandulaire ; r. : ribosomes.

Dimensions : Mâle allotype : corps long de $30 \mathrm{~mm}$ et large de $500 \mu \mathrm{m}$; anneau nerveux à $150 \mu \mathrm{m}$ de l'apex; œsophage long de 5,7 mm et portion glandulaire débutant à $230 \mu \mathrm{m}$ de l'apex ; spicule gauche long de $5,7 \mathrm{~mm}$; spicule droit long de $140 \mu \mathrm{m}$; queue longue de $55 \mu \mathrm{m}$.

Mâle paratype : corps long de $33 \mathrm{~mm}$ et large de $450 \mu \mathrm{m}$; anneau nerveux à $190 \mu \mathrm{m}$ de l'apex; œsophage long de $5,5 \mathrm{~mm}$; spicule gauche cassé ; spicule droit et queue longs de 140 et $150 \mu \mathrm{m}$.

\section{Discussion}

La Filaire du Dermoptère est à rapprocher de deux Dirofilariinae de singes orientaux, Edesonfilaria malayensis Yeh, 1960 et Macacanema formosana Schad et Anderson, 1963, et d'une Filaire parasite de Megachiroptère en Malaisie, Makifilaria 
inderi Krishnasamy et coll., 1981, qui a été placée dans les Lemdaninae, près d'un autre parasite de Chauve-souris, Chiropterofilaria Yeh et coll., 1958. En fait les dimensions et les figures données par Krishnasamy et coll. montrent qu'il s'agit d'une espèce très proche d'E. malayensis; le genre Makifilaria n'est donc pas nécessaire et peut être placé en synonymie d'Edesonfilaria.

Ces Filaires ont en commun la grande taille du corps, la queue courte et arrondie, les spicules très inégaux, la présence de papilles caudales surnuméraires alignées en avant du cloaque et l'œsophage de structure caractéristique.

La portion musculaire comprend une partie antérieure libre et une partie postérieure d'environ $1 \mathrm{~mm}$ de long, accolée à la portion glandulaire et qui s'atténue au fur et à mesure jusqu'à disparaître. La portion glandulaire est exceptionnellement longue (5 à $90 \mathrm{~mm}$ ) et remarquable aussi par son aspect sombre dû à la présence de très nombreux granules; elle a été interprétée initialement comme un sac à paroi mince et à lumière très grande remplie de "grains de sécrétion " et bordé d'organes énigmatiques, les " giantcells " ou " giant bodies " (Yamaguti et Hayama, 1961 ; Schad et Anderson, 1963) ; il apparaît maintenant avec la Filaire du Cynocephale que l'œsophage glandulaire est un syncitium géant, aux noyaux volumineux disposés à la périphérie (= " giant cells " ou " giant bodies ") et au cytoplasme chargé de ribosomes (= "grains de sécrétion ») ; cette portion glandulaire est dépourvue de lumière, les substances ingérées subissent vraisemblablement dans cet organe une transformation sous l'action des ribosomes.

La Filaire du Dermoptère s'oppose aisément à Macacanema formosana par la forme différente du spicule, filiforme et long de $11 \mathrm{~mm} \mathrm{chez} \mathrm{nos} \mathrm{spécimens,} \mathrm{avec}$ lame et manche distincts et long de $590 \mu \mathrm{m}$ chez $M$. formosana. En outre cette dernière espèce a une plus grande taille, un œsophage plus long et des ailes caudales bien marquées.

La morphologie du spicule rapproche par contre la Filaire du Dermoptère des deux espèces d'Edesonfilaria.

E. malayensis est distinct de nos spécimens par la plus grande taille du corps et de l'œsophage, les ailes caudales bien visibles, le spicule gauche deux fois plus long, les papilles caudales surnuméraires plus nombreuses (10 paires au lieu de 2 à 4 paires) et la microfilaire plus petite $(140 \mu \mathrm{m} / 2,5$ au lieu de $190-212 \mu \mathrm{m} / 3,5 \mu \mathrm{m})$.

E. inderi est distinct par la taille plus grande de la femelle, le spicule droit plus court et la microfilaire plus courte $(90-170 \mu \mathrm{m})$.

La Filaire du Dermoptère constitue donc une nouvelle espèce, Edesonfilaria cynocephali $\mathrm{n}$. sp.

\section{Conclusion}

La Filaire du Cynocéphale, E. cynocephali n. sp., celle du Pteropus, E. inderi, et les 2 espèces parasites des Macaques, $E$. malayensis et $M$. formosana constituent un petit groupe naturel de Filaires, aux ailes caudales peu marquées et à l'œsophage hyperspécialisé. Placé actuellement dans les Dirofilariinae, il conviendrait peut-être mieux de le reclasser dans les Lemdaninae. 
Cette petite lignée, parasite de Dermoptères, Chiroptères et Primates arboricoles, est propre à la région indo-malaise.

L'espèce parasite de Macaca cyclopsis à Taiwan, M. formosana peut être considérée comme la plus primitive avec un spicule gauche encore peu allongé, à manche et lame distincts. Les trois autres espèces, originaires de Malaisie et de Thaillande, se groupent dans le genre Edesonfilaria plus évolué, au spicule très long et filiforme, et sont proches les unes des autres.

\section{BIBLIOGRAPHIE}

Krishnasamy M., Mulkit Singh, Rajoo Iyamperumal : Makifilaria inderi gen. et sp. nov. (Filarioidea : Onchocercidae) from the island flying fox, Pteropus hypomelanus Temminck in Malaysia. Southeast Asian J. Trop. Med. Pub. Heth, I981, 12, I85-188.

Schad G. A., Anderson R. C. : Macacanema formosana n. g., n. sp. (Onchocercidae : Dirofilariinae) from Macaca cyclopsis of Formosa. Canad. J. Zool., 1963, 4I, 797-80o.

Yamaguti S., Hayama S. : A redescription of Edesonfilaria malayensis Yeh, I960, with remarks on its systematic position. Proc. Helm. Soc. Washington, I961, 28, 83-86.

YEH L.-S. : On a new filarioid worm, Edesonfilaria malayensis gen. and sp. nov. from the longtailed Macaque (Macaca irus). J. Helm., 1960, 34, 125-128. 